\title{
DEVELOPMENT OF SYNAPSES IN THE ANTENNAL LOBES OF THE MOTH MANDUCA SEXTA DURING METAMORPHOSIS ${ }^{1}$
}

\author{
L. P. TOLBERT ${ }^{*, 2}$ S. G. MATSUMOTO, ${ }^{*}$ AND J. G. HILDEBRAND $\ddagger^{3}$ \\ * Department of Neurobiology, Harvard Medical School, Boston, Massachusetts 02115 and $\ddagger$ Department of Biological \\ Sciences, Columbia University, New York, New York 10027
}

Received September 29, 1982; Revised December 27, 1982; Accepted December 28, 1982

\begin{abstract}
During the metamorphosis of holometabolous insects, the larval nervous system is restructured to provide the circuitry needed by the developing adult. Prominent new centers in the brain, the antennal lobes, arise to receive olfactory afferent axons from the developing adult antennae and provide an excellent system in which to study the development of synapses in a central nervous system. We have examined the anatomy and physiology of developing synapses in the antennal lobes of the moth Manduca sexta during the 18 days of metamorphic adult development. On day 5, the neuropil of the newly emerging antennal lobe condenses into distinct glomeruli, in which intercellular junctional complexes have already begun to form. Although some junctions have associated synaptic vesicles, most complexes are desmosome-like until day 9 , when the number of synaptic complexes begins to increase. Early synapses are characterized by membrane-associated densities in at least two abutting cellular processes and a small number of synaptic vesicles clustered near the membrane of one process. As adult development proceeds, the membrane-associated densities become denser and more extensive, and the number of synaptic vesicles in the clusters increases. At day 14 synapses appear ultrastructurally mature, and almost all junctions in the neuropil can be identified as synaptic. Not until day 9 do antennal lobe neurons begin to respond postsynaptically when the antennal nerve is stimulated electrically, suggesting that the earliest synapses observed in the electron microscope may not be made by antennal nerve axons. At first the postsynaptic responses are graded and fatigue rapidly. By day 11, the antennal lobe neurons respond with action potentials, but the fatigability does not decline to adult levels until day 13 . Filling of antennal lobe neurons with cobalt reveals that the arborizations of both local interneurons and output neurons continue to mature morphologically until about day 13. Previous work (Schweitzer, E. S., J. R. Sanes, and J. G. Hildebrand (1976) J. Insect Physiol. 22: 955-960) showed that antennal sensilla do not begin to be responsive to odors until day 14. Thus the establishme it of the synaptic network in the antennal lobe apparently occurs in the absence of functional olfactory input to the system.
\end{abstract}

Neurons in vertebrates often depend upon establishment of contact with their targets for their survival and

${ }^{1}$ This work was supported by National Science Foundation Grants BNS-77-13281 and BNS-80-13511 and by National Institutes of Health Grant AI-17711. L. P. T. and S. G. M. were supported by National Institutes of Health postdoctoral fellowships and National Institutes of Health Training Grant NS-07112. We thank M. Imperato and R. Montague for excellent technical assistance, $\mathrm{H}$. Harrow and J. Torrisi for secretarial help, A. M. Schneiderman for collaboration on deafferentation experiments, Drs. A. H. Baumhover, J. A. Svoboda, and C. M. Williams for supplying Manduca eggs, and Dr. A. Fröhlich for helpful comments on the manuscript.

${ }^{2}$ Present address: Department of Anatomy, Georgetown University School of Medicine, 3900 Reservoir Road, Washington, DC 20007.

${ }^{3}$ To whom correspondence should be addressed at Department of Biological Sciences, Fairchild 913, Columbia University, New York, NY 10027. normal maturation (e.g., Cowan and Wenger, 1967; Black et al., 1972; Landmesser and Pilar, 1972) and may strongly influence the development of their target cells (e.g., Guth, 1957; Black et al., 1971). Invertebrate neurons, such as the lamina monopolar cells in the firstorder optic neuropil of several arthropods (e.g., Kopéc, 1922; Macagno, 1977; Anderson, 1978; Maxwell and Hildebrand, 1981), likewise may depend upon appropriate cell-cell contacts for their normal development.

In the olfactory pathway of the moth Manduca sexta, however, the effects of pre- or postsynaptic deprivation seem to be less drastic. The sensory neurons in the antennal flagellum follow the normal schedule of morphological, biochemical, and electrophysiological differentiation even when deprived of their target cells in the brain (Sanes et al., 1976). Moreover, the antennal lobe, the primary olfactory center in the brain, when deprived 
of its antennal afferent input, develops some but not all of its normal structural and neurochemical characteristics (Sanes et al., 1977; Hildebrand et al., 1979). Although the synaptic neuropil of the normal antennal lobe comprises an orderly array of discrete glomeruli, the neuropil of a lobe deprived of antennal afferent input throughout adult development is significantly altered and resembles that of a normal lobe arrested at an early stage of development. This finding suggests that the incoming sensory axons play an important role in the organization of the neuropil of the antennal lobe.

This idea gains support from studies of sexually dimorphic elements in the olfactory pathway in Manduca. The synaptic neuropil of each normal male antennal lobe includes a large macroglomerular complex which contains arborizations of male-specific neurons that receive afferent input from male-specific, pheromone-sensitive antennal sensory neurons and is not present in normal female antennal lobes (Matsumoto and Hildebrand, 1981). Ingrowing sensory axons from a male antenna are required for formation of this sexually dimorphic neuropil structure, and a male antenna, grafted at an early developmental stage onto a female host, can induce or permit the development of the macroglomerular complex and its associated male-specific interneurons in the host's antennal lobe (Schneiderman et al., 1982).

Thus it seems that intercellular regulation takes place during the development of the antennal lobe in Man$d u c a$, but more selectively than in many other systems. Because the opportunities for intercellular regulation may be limited, the antennal lobe of Manduca promises to be a good model system in which to probe the mechanisms underlying this important kind of cell-cell interaction.

The development of the adult antennal system proceeds during metamorphosis, when the larva is transformed into the adult by way of a pupal stage. At this time in postembryonic life, when the animal has attained large size, the developmental stages are experimentally accessible and easily studied. The adult antennae develop from antennal imaginal disks, as has been described in detail (Sanes and Hildebrand, 1976 a, b). The development of the antennal lobes of the brain, however, has been less thoroughly explored.

As a first step toward understanding the role of antennal sensory neurons in regulating the development of their targets in the antennal lobes, we have examined the normal sequence of developmental events in the lobes of Manduca. In particular we have focused on the temporal relationships between the ingrowth of primary afferent fibers, the extension of neurites by the neurons of the antennal lobe, and the physiological and ultrastructural maturation of synapses in the lobe, and we have compared this developmental timetable with that derived by Sanes and Hildebrand $(1976 a, b)$ for the antennal sensory neurons. This study is a necessary first step toward an investigation of mechanisms governing the development of the antennal lobes, as it provides constraints for future hypotheses and guidance for the design of experiments.

\section{Materials and Methods}

Animals. Manduca sexta (Lepidoptera: Sphingidae) were reared as previously described (Sanes and Hilde- brand, 1976a). Adult development, triggered at the time of the larval-pupal molt, normally occurs over 18 staged "days" and ends with the eclosion of the adult moth 19.8 \pm 1.3 days after pupal ecdysis (Sanes and Hildebrand, 1976a). The developmental stage of a metamorphosing animal was determined on the basis of morphological changes of the cuticle or of structures visible through the cuticle. "Day 1 " is defined as the stage that occurs during roughly the first $24 \mathrm{hr}$ of adult development, "day 2", the next $24 \mathrm{hr}$, and so on. The percentage of adult development represented by each staged day is presented in Table I.

In order to deprive the developing brain of antennal input, antennal imaginal disks were removed from fifth instar larvae according to the procedure of Schneiderman et al. (1982), thus preventing the development of adult antennae.

Histology. Each animal was cooled on ice, and the brain, moistened with physiological saline solution, was excised. The brain was fixed in modified Serra's fixative solution (Nair, 1963), dehydrated, and embedded in Paraplast Plus (Sherwood Medical Industries, St. Louis, MO). Ten-micron sections were cut, stained with Cresylecht violet and Luxol fast blue (Luna, 1968), dehydrated, and mounted in Permount.

Intracellular recording and staining. For intracellular recording and staining of neurons in the antennal lobe, we used our previously published methods (Matsumoto and Hildebrand, 1981). All intracellular recordings were obtained with glass microelectrodes filled with a solution of $158 \mathrm{~mm} \mathrm{CoCl}_{2}$ and $0.75 \mathrm{M} \mathrm{KCl}$. Following injection of cobalt with depolarizing current pulses (5 to $10 \mathrm{nA}$ ), the preparations were treated with ammonium sulfide and intensified with silver as described before (Bacon and Altman, 1977; Matsumoto and Hildebrand, 1981). The

TABLE I

Summary of preparations examined

\begin{tabular}{|c|c|c|c|c|}
\hline \multicolumn{2}{|c|}{ Developmental Stage } & \multicolumn{2}{|c|}{$\begin{array}{l}\text { Intracellular Recording and } \\
\text { Staining }\end{array}$} & \multirow{2}{*}{$\begin{array}{l}\text { Electron Mi- } \\
\text { croscopy }^{d}\end{array}$} \\
\hline Staged "Day" & $\begin{array}{l}\text { Approximatt } \\
\text { Percent of Adult } \\
\text { Development }\end{array}$ & $\begin{array}{l}\text { Type-1b Lo- } \\
\text { cal Interneu- } \\
\text { rons }^{b}\end{array}$ & $\begin{array}{l}\text { Type-1 Out- } \\
\text { put Neurons }\end{array}$ & \\
\hline 5 & $23-29$ & & & 2 \\
\hline 6 & $29-35$ & & & 1 \\
\hline 7 & $35-41$ & & & 4 \\
\hline 8 & $41-47$ & $5(0)$ & & $3(1)$ \\
\hline 9 & $47-53$ & $23(8)$ & 5 & 5 \\
\hline 10 & $53-59$ & & 8 & 4 \\
\hline 11 & $59-65$ & $30(12)$ & & $4(1)$ \\
\hline 12 & $65-71$ & & 3 & $8(2)$ \\
\hline 13 & $71-76$ & $15(5)$ & 3 & 4 \\
\hline 14 & $76-82$ & & & 3 \\
\hline 15 & $82-88$ & $28(12)$ & 1 & \\
\hline 16 to adult & $88-100$ & $55(20)$ & & $13(1)$ \\
\hline
\end{tabular}

${ }^{2}$ Not including neurons reported previously (Matsumoto and Hildebrand, 1981).

${ }^{b}$ The first number refers to the total number of cells recorded from; the number in parentheses specifies the number of those cells that were also stained with cobalt sulfide.

"As explained under "Materials and Methods," these cells were stained but not studied physiologically.

${ }^{d}$ The first number gives the total number of animals studied ultrastructurally; the number in parentheses specifies the number of those animals that had been deantennated. 
preparation was cleared in methyl salicylate and then either photographed directly or, when finer detail was required, drawn with the aid of a camera lucida.

Antennal sensory axons in developing animals were stimulated electrically by means of a pair of extracellular hook electrodes placed around the antennal nerve about 1 to $2 \mathrm{~mm}$ from its entrance into the antennal lobe. These bipolar electrodes were fashioned from Teflon-insulated silver wires ( $100 \mu \mathrm{m}$ in diameter, Medwire). The Teflon insulation was scraped off to expose the wires where they contacted the antennal nerve. The electrodes were further insulated from the surrounding saline bath by covering them with a layer of petroleum jelly. Brief pulses of current $(0.5$ to $2 \mathrm{msec})$ were delivered using a WPI digital stimulator. The stimulus was adjusted to a level at which further increases in its strength no longer changed the response recorded from the antennal lobe neuron under study. This was taken to be the stimulus intensity at which a maximal recruitment of sensory axons had been obtained.

Specimens were examined physiologically and morphologically at various stages of development complementing those used for electron microscopy (Table I). Our recording procedures proved to be inadequate to obtain reproducible results prior to day 8 of adult development because the brain is extremely fragile at early stages of metamorphosis. The most immature preparations examined were the least amenable to intracellular recording and staining, because these neurons were generally difficult to impale for the minimal time (15 to 20 min) required to test a neuron physiologically and to inject it with cobalt.

Electron microscopy. Each animal was cooled on ice, and the brain was quickly removed and fixed and stained according to one of two procedures.

For optimal ultrastructural preservation, the brain was fixed by immersion in ice-cold fixative solution containing $0.5 \%$ paraformaldehyde, $2.5 \%$ glutaraldehyde, $0.18 \mathrm{mM}$ $\mathrm{CaCl}_{2}, 0.58 \mathrm{mM}$ sucrose, and $0.1 \mathrm{M}$ sodium cacodylate buffer, pH 7.4 (modified from Karnovsky, 1965), and then processed as described previously (Tolbert and Hildebrand, 1981). This procedure was effective in pupae as young as day 5 . We were unable to obtain adequate fixation in younger animals, however, using this or other aldehyde fixatives.

The procedure of Simionescu et al. (1972) for visualizing tracer carbohydrate moieties was used in some cases specifically to enhance the contrast of membrane-associated junctional densities. In this procedure the brain is simultaneously fixed, osmicated, and stained with lead by immersion in an ice-cold solution containing paraformaldehyde, glutaraldehyde, $\mathrm{OsO}_{4}$, and lead citrate in phosphate buffer, for $2 \mathrm{hr}$. The brain is then dehydrated as quickly as possibly and embedded in Epon. In contrast to the phosphotungstic acid (Bloom and Aghajanian, 1968) and bismuth iodide-uranium-lead (Pfenninger, 1971) methods, this procedure results in good overall ultrastructural preservation as well as enhanced junctional staining and was, therefore, more useful than either of the other two methods investigators have used to visualize membrane-associated densities. Because this method is very sensitive to the age and composition of the stock lead solution, we inspected glycogen granules, which are concentrated in glial cells, in every preparation in order to ensure that we used only those in which carbohydrate-specific staining had occurred. In animals younger than day 10 , the method produced highly variable qualities of fixation and was, therefore, not used.

Thin sections of the Epon-embedded antennal lobes were picked up on clean or Formvar-coated grids. Sections from the lead-treated blocks of tissue were stained only with lead citrate (Venable and Coggeshall, 1965); sections from material fixed by the first method were stained with either lead citrate alone or both $1 \%$ uranyl acetate (in water or $50 \%$ methanol) and lead citrate. Sections were examined in a Philips 400 electron microscope.

\section{Results}

\section{Histological appearance of the antennal lobe through metamorphosis}

During the first 4 days of adult development, each antennal lobe appears in sections stained with Cresylecht violet and Luxol fast blue as a small mound of homogeneous neuropil bounded by cell bodies (Fig. 1a). The antennal lobes sit on the anterior surface of the brain where the antennal nerves enter the deutocerebrum dorsally. By day 5, outer regions of neuropil have begun to condense and subdivide into discrete, differentially staining glomeruli, and the neuronal cell bodies have become segregated into clusters (Fig. 1b). At this and later stages, we refer to the neuropil within glomeruli as "glomerular neuropil." During the next day, the subdivision of neuropil into spheroidal glomeruli is completed, and glial cells begin to form a partial shell around each glomerulus (Fig. 1c). After day 6, the antennal lobes continue to grow in overall dimensions, and the glomeruli grow to occupy proportionately more of the lobe. On day 9 the antennal lobes shift dorsally to occupy their adult positions on the anterodorsal surface of the brain, and on day 10 the central neuropil becomes coarser in texture, owing to the presence of large, crisscrossing neurites. By day 13 , the antennal sobe is light microscopically indistinguishable in size and character from that of the adult (Fig. 1d).

\section{Development of second-order neurons: electrophysiology and morphology}

A number of distinct physiological and morphological classes of neurons have been described in the antennal lobe of adult Manduca (Matsumoto and Hildebrand, 1981). In the previous study many of these classes were distinguished on the basis of their responses to chemical stimuli delivered to the antennae. We have found that some of the types of antennal lobe neurons can also be recognized from their responses to electrical stimulation of the antennal nerve. We used such electrical stimulation of antennal afferents in this developmental study because olfactory reception in the antennae matures too late to be useful for monitoring maturational changes in the antennal lobes (Schweitzer et al., 1976).

The simplest type of response evoked in an antennal lobe neuron by a single suprathreshold shock to the 

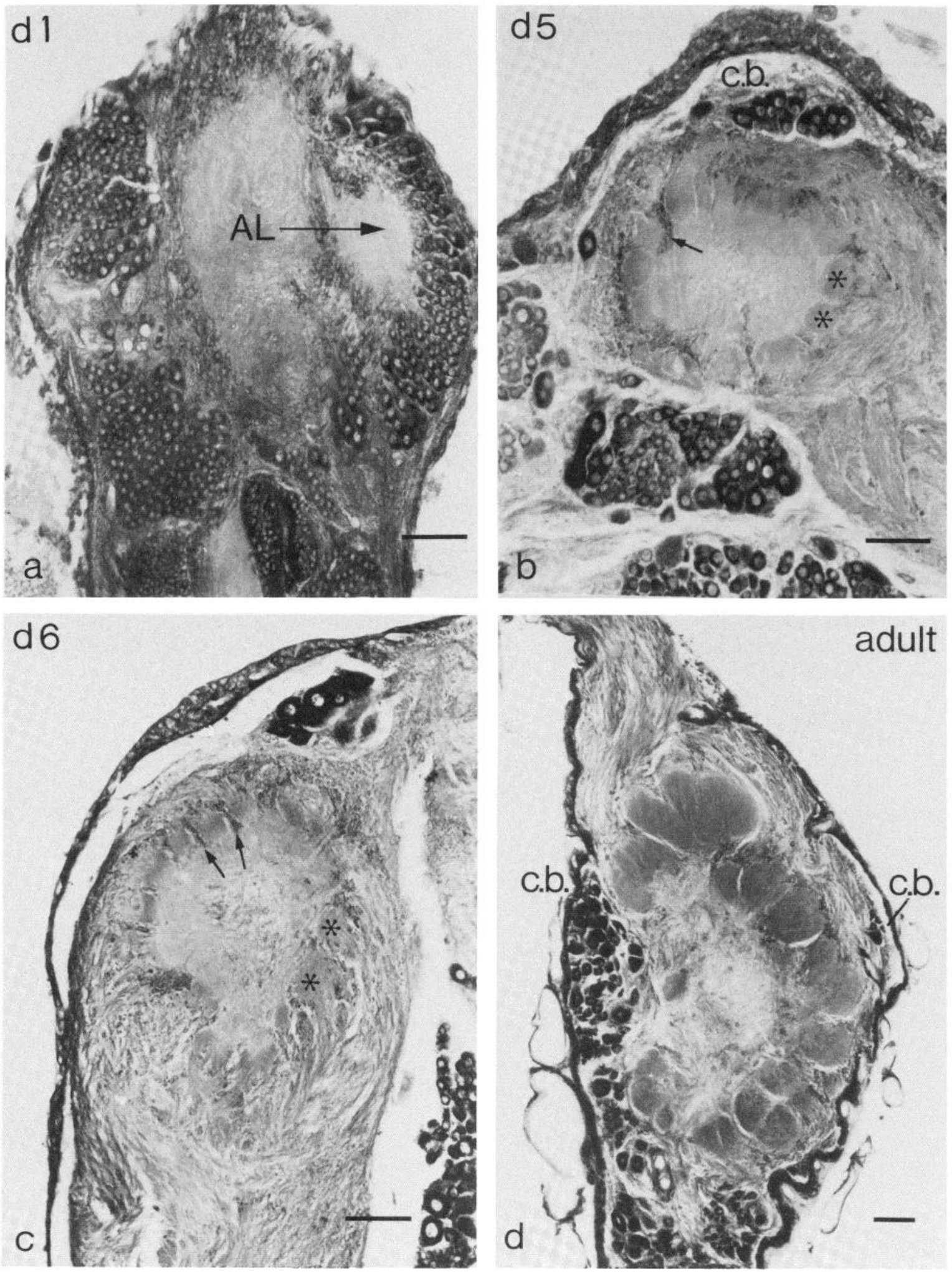

Figure 1. Light micrographs of sections stained with Cresylecht violet and Luxol fast blue through antennal lobes at different stages of adult development. All scale bars equal $100 \mu \mathrm{m}$. $a$, Day 1 (d1) animal, horizontal section through brain. $A L$, antennal lobe, comprising homogeneous neuropil surrounded by cell bodies. $b$, Day $5(d 5)$ animal, oblique section. Neuronal cell bodies are now in two large clusters, one of which appears at top (c.b.). Neuropil exhibits spheroidal glomeruli (*), some of which are delimited by glial cells (e.g., at arrow). c, Day $6(d 6)$ animal, transverse section. Glial cells (arrows) now insinuate between many glomeruli (*). The central neuropil has become coarser. $d$, Adult moth, frontal section. Note change in scale. The antennal nerve enters from above. Glomeruli are large and stain darkly. A large lateral cluster of cell bodies and a small medial cluster of cell bodies (c.b.) are visible. 
antennal nerve is very frequently observed: a monophasic depolarizing synaptic potential, accompanied by a brief burst of action potentials. This response has a short latency ( $<10 \mathrm{msec}$ ) and declines gradually with repeated stimulation. Both of these characteristics suggest that the pathway is monosynaptic, but technical difficulties have impeded more powerful pharmacological or anatomical testing of this idea. Intracellular staining of cells exhibiting this type of response reveals them to be local interneurons with multiglomerular arborizations that arise in a radially symmetric pattern from the primary neurite. These neurons, classified as type-Ib local interneurons by Matsumoto and Hildebrand (1981), are called local interneurons in this paper in the interest of simplicity.

Other, more complex types of responses, involving longer latency excitatory or inhibitory and excitatory postsynaptic potentials, are also observed, but none of these can be ascribed consistently to one morphological class of neuron. In the present study, we have concentrated primarily on the development of the local interneurons because of the relative simplicity of their response to electrical stimulation of antennal sensory axons and the reliability with which the physiological response of these cells can be correlated with a morphological type. We have also examined the morphological development of a class of antennal lobe output neurons.

Physiology. On day 8, when all of the ingrowing sensory axons from the antennal flagellum have reached the base of the antenna (Sanes and Hildebrand, 1976b) and most have entered the antennal lobe (Camazine and Hildebrand, 1979), no synaptic potentials are detectable in any of the antennal lobe neurons following stimulation of the afferent axons. Although numerous sensory axons were activated by our stimulus conditions at this stage of development, we cannot be confident that the entire population of afferent fibers can conduct action potentials on day 8 . The central neurons exhibit the normal range of resting potentials $(50$ to $70 \mathrm{mV}$ ) and are capable of generating action potentials at this time, as evidenced by a burst when the neuron is initially impaled by a microelectrode or when depolarizing current is passed into the cell (not shown).

Synaptic transmission is first detected in the local interneurons on day 9 of adult development (Fig. 2, first column). At this time only subthreshold synaptic potentials are observed when the antennal nerve is stimulated electrically, even though, as earlier, the resting potential of the neurons and their ability to conduct action potentials are within the ranges normally observed at later developmental stages. Synaptic transmission at this early stage is susceptible to fatigue. The postsynaptic potential recorded in the interneuron rapidly diminishes when the antennal nerve is stimulated at $0.5 \mathrm{~Hz}$. At the end of the thirticth stimulus in the train, no postsynaptic potentials can be detected. Full recovery of the response occurs in approximately $5 \mathrm{~min}$. None of the more complex types of responses, presumably mediated by as yet immature polysynaptic pathways, were detected on day 9 .

Synaptic efficacy increases dramatically 1 to 2 days later (day 10 to 11). Recordings from interneurons at this stage reveal all of the types of responses recognized in adult moths. Specifically, the response of the local inter- neurons to single afferent stimuli resembles that of the adult neurons (compare top traces, Fig. 2). The susceptibility to synaptic fatigue is still present, however (Fig. 2 , second column), although not to the extent observed at day 9 .

Recordings from the local interneurons on day 13 and thereafter revealed physiological characteristics indistinguishable from those of their adult counterparts, with no sign of fatigue at moderate rates of stimulation (Fig. 2, third column).

Morphology. The physiologically characterized local interneurons were visualized by intracellular staining. The adult local interneurons have a multiglomerular arborization that arises in a radially symmetrical manner from a single, large central neurite (Fig. $3 d$; see also Figs. 3 and 4 in Matsumoto and Hildebrand, 1981). In addition, branching neurites within the glomeruli are covered with spicules.

The youngest local interneurons successfully stained were obtained at day 9, when many of the features of this type of neuron are evident. The neurites have already grown widely into the developing glomerular neuropil, and the neuron's characteristically radial branching pattern is clearly present (Fig. $3, a$ and $b$ ). The branching of the neurites in the glomeruli is sparse, however, and the spicules that cover the neurites of the mature neuron are absent.

At day 11 to 12 , all of the features of the mature neuron, including the spicules, have appeared (Fig. 3c). Qualitatively, however, it is clear that the glomerular branching and the distribution of spicules are less dense than observed in fully developed neurons. Thus it appears that both the physiological properties of synaptic transmission and the morphological characteristics of the neuron are at an intermediate stage of development until day 13 , when the morphology-like the physiology-of all of the local interneurons examined is indistinguishable from that of mature neurons (Fig. $3 d$ ).

In this study we also examined the morphological development of a class of output neurons. These cells, previously described as type-I output neurons (see Figs. 20 and 21 in Matsumoto and Hildebrand, 1981), are simply called output neurons in this paper. Unfortunately, the physiological response of the mature output neuron to single electrical shocks delivered to the antennal nerve varies considerably from neuron to neuron. Thus it has not been possible to study the physiological development of these cells using the same methods employed for the local interneurons. The mature output neuron has several distinguishing morphological features (Matsumoto and Hildebrand, 1981). Its arborization in the glomerular neuropil is always confined to a single glomerulus; the neurites branch in a nonuniform manner, sparsely within the inner hemisphere and very densely in the outer hemisphere of the glomerulus. The neuron always has an axon projecting out of the antennal lobe into the protocerebrum. At day 9 many of these features are present (Fig. 4, $a$ and $b$ ). A distinct but sparse uniglomerular tuft of branching neurites has formed, and the nonuniformity of the glomerular arborization of the adult neuron is evident. The axon has reached the protocerebrum. The output neurons identificd at this stage also show a number of thin, sparsely branching neurites 


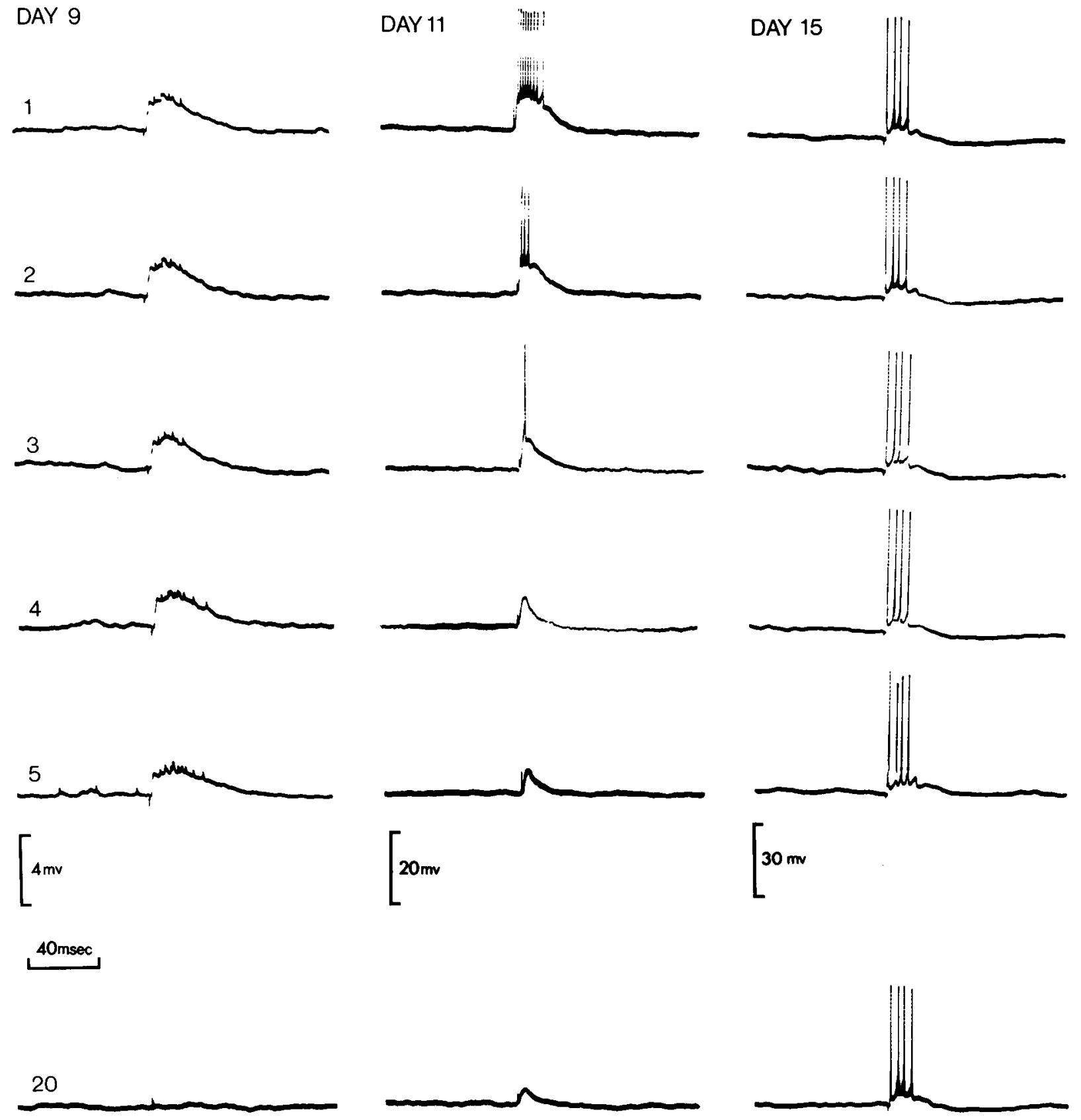

Figure 2. Response of the local interneurons to repeated electrical stimulation of the antennal nerve at $0.5 \mathrm{~Hz}$. Each column shows the response of the neuron in an animal of the developmental stage indicated at the top. The first five rows display the responses observed to the first five stimuli in the train. The last row gives the response to the twentieth stimulus. Day 9, Only subthreshold responses are observed. Repeated stimulation results in a rapid decrease in amplitude of the postsynaptic potential Day 11, Suprathreshold responses are elicited by the first three presentations of the stimulating shock, but the response decreases rapidly with repeated stimuli. Day 15, The response is typical for the local interneurons from day 13 to adult. The response of the neuron is constant at this low rate of stimulation. Note the difference between the vertical calibrations of the three examples.

extending into other regions of the antennal lobe neuropil, a pattern that has never been observed in any neurons of this class in mature antennal lobes. At a later stage of development (day 11 to 12 ), the uniglomerular neuritic tuft branches extensively (as in the adult), and all but a few of the "extraglomerular" processes are absent (Fig. $4 c$ ). By day 13 all of the output neurons observed appear to have matured morphologically.

\section{Ultrastructural maturation of the antennal lobe}

Organization of the mature antennal lobe. In the adult moth, the cell bodies of neurons, clustered in pack- ets at the edge of the antennal lobe, are separated from each other by several layers of glial processes. All of the ultrastructurally recognized chemical synapses in the mature antennal lobe are in the glomeruli, of which only the "ordinary," spheroidal glomeruli found in both males and females (Matsumoto and Hildebrand, 1981) have been examined in this study. Most presynaptic profiles are filled with either round, clear vesicles $(25$ to $30 \mathrm{~nm}$ in diameter) or numerous large dense-cored vesicles $(>45$ $\mathrm{nm}$ in diameter) accompanied by round, clear vesicles (Tolbert and Hildebrand, 1981). A few profiles contain a population of clear, pleiomorphic vesicles. At each syn- 

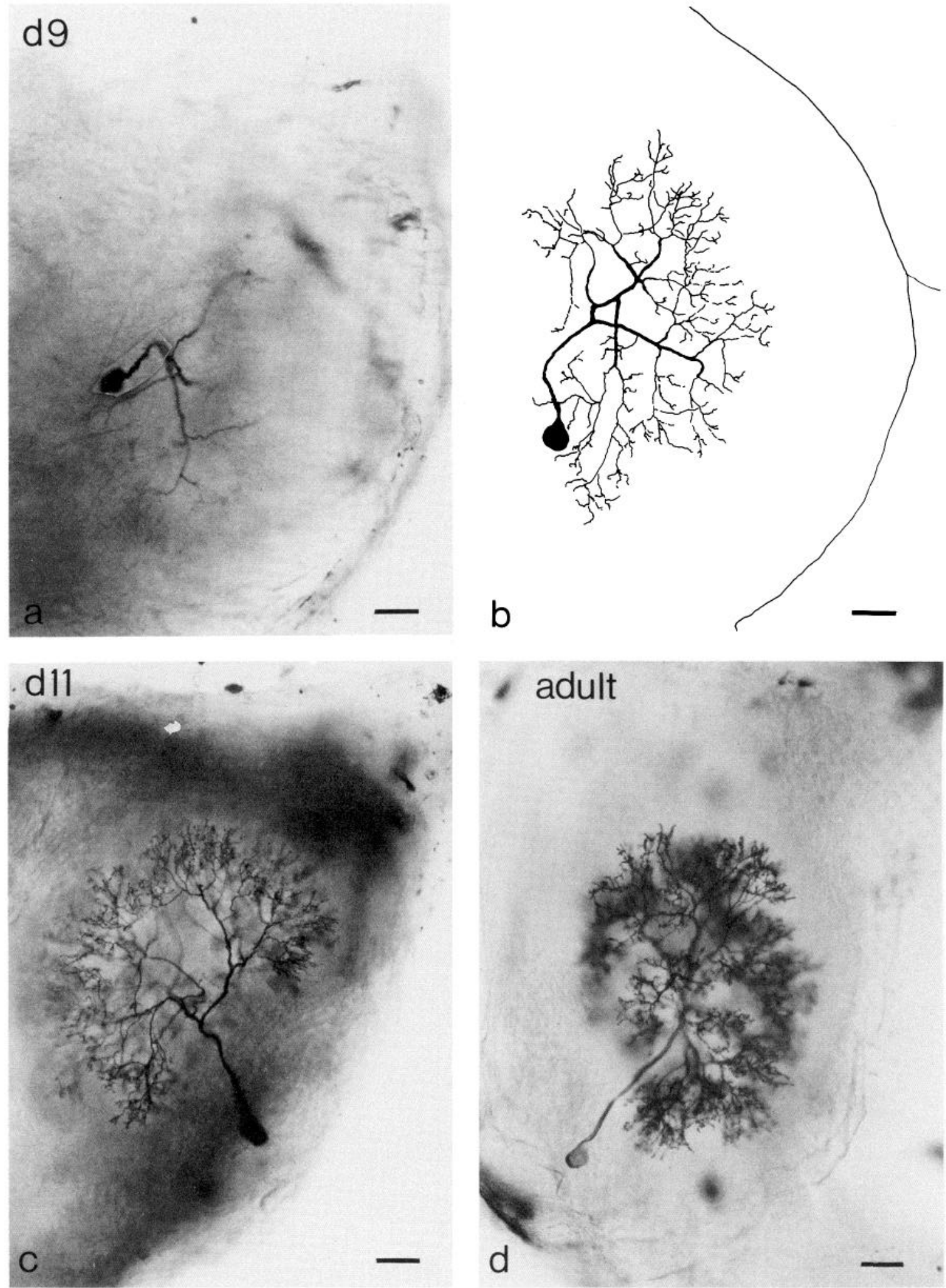

Figure 3. Photomicrographs and drawing of whole mounts illustrating stages in the morphogenesis of the local interneurons. Each neuron was injected with cobalt and intensified with silver as described under "Materials and Methods." All scale bars equal $100 \mu \mathrm{m}$. $a$, Day $9(d 9)$, montage of two photomicrographs taken at different planes of focus. The arborization of the neurites is sparse, and their surfaces are smooth. $b$, Camera lucida drawing of the neuron shown in $a$. $c$, Day 11 (d11), the glomerular processes of the neurites branch more elaborately and are lightly studded with spicules. $d$, Day 15 , mature neuron with dense neuritic branching in the glomerular neuropil. Terminal branches are covered with spicules. 

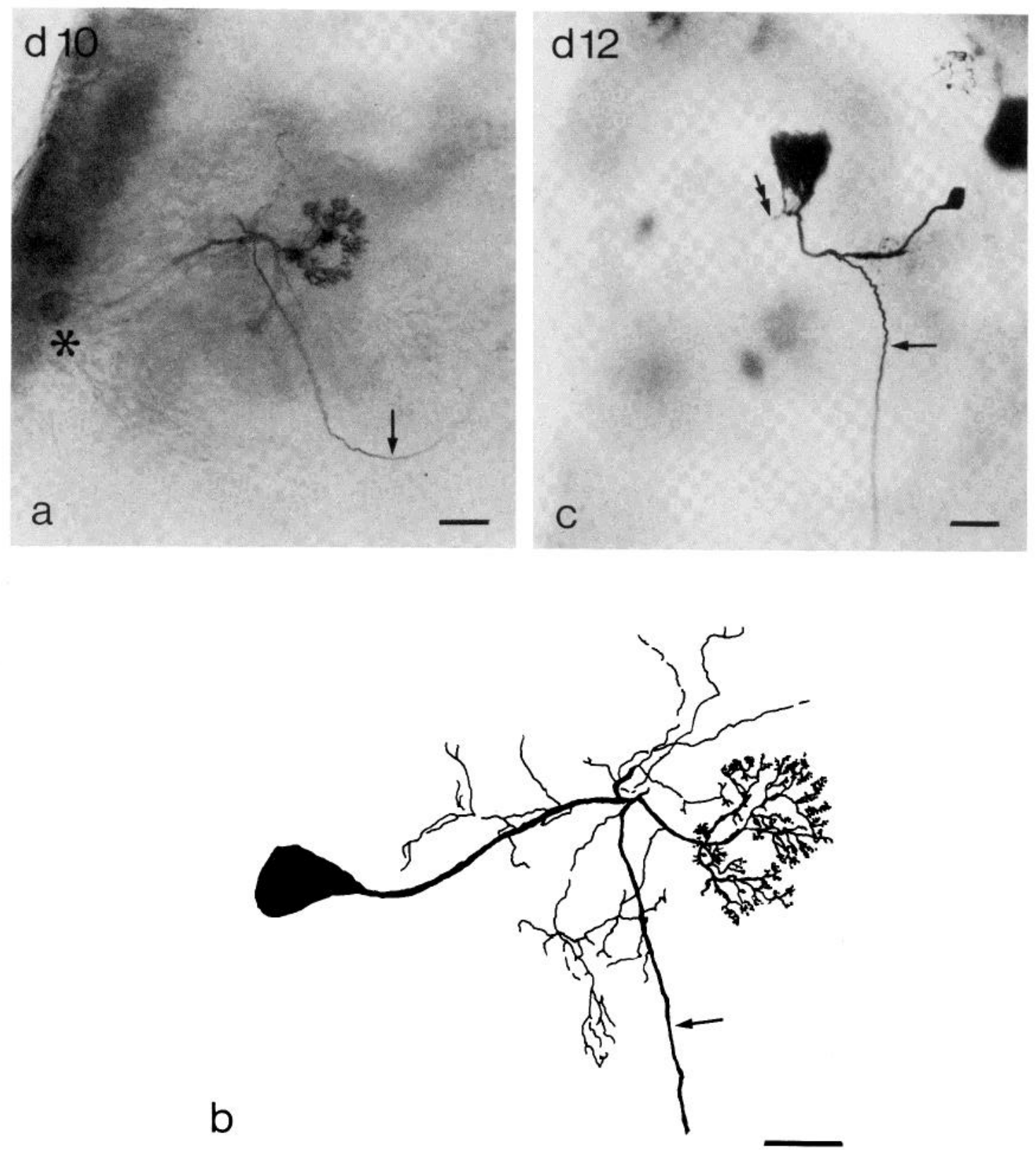

Figure 4. Photomicrographs and drawing of whole mounts illustrating stages in the morphological development of the output neurons. Each neuron was injected with cobalt and intensified with silver as described under "Materials and Methods." In each case the axon is indicated with an arrow. All scale bars equal $100 \mu \mathrm{m}$. $a$, Day 10, at this stage many neurites extend into the glomerular neuropil. One of them is thicker and branches more elaborately than the others. The cell body (*) is stained lightly. The neuron sends an axon out of the antennal lobe. $b$, Camera lucida drawing of the neuron shown in $a$. $c$, Day 12 , this neuron has all of the morphological features of a mature example, except that a few small processes (one at double arrow:) extend outside of the glomerular neuritic tuft, a condition not ordinarily observed in mature neurons.

aptic complex, a presynaptic element contacts multiple postsynaptic elements by pairs. The presynaptic density is bar-shaped (up to $0.5 \mu \mathrm{m}$ long) and flanked by rows of synaptic vesicles, and postsynaptic membranes have wispy densities. No junctions other than synapses have been unequivocally identified in the glomerular neuropil of the mature antennal lobe.

Days 5 through 8 . On days 5 through 8 of adult development, when antennal axons are growing into the antennal lobe (Camazine and Hildebrand, 1979) and the neuropil has just become organized into glomeruli, the lobe is ultrastructurally immature. The cell bodies in the medial and lateral clusters of neurons are closely packed, separated only by a sometimes discontinuous network of slender glial processes. In the lacunae between glial processes, the plasma membranes of adjacent neurons may be closely apposed, with little or no intervening space (Fig. $5 a$ ). They do not have the rigid regularity of specialized junctional membranes (e.g., at gap junctions), but do appear here and there to touch (Fig. $5 a$, inset). It is possible that tight junctions are formed; freeze-fracture of the cell bodies will be necessary to establish the nature of the contacts.

At this stage, the texture of the glomerular neuropil is 

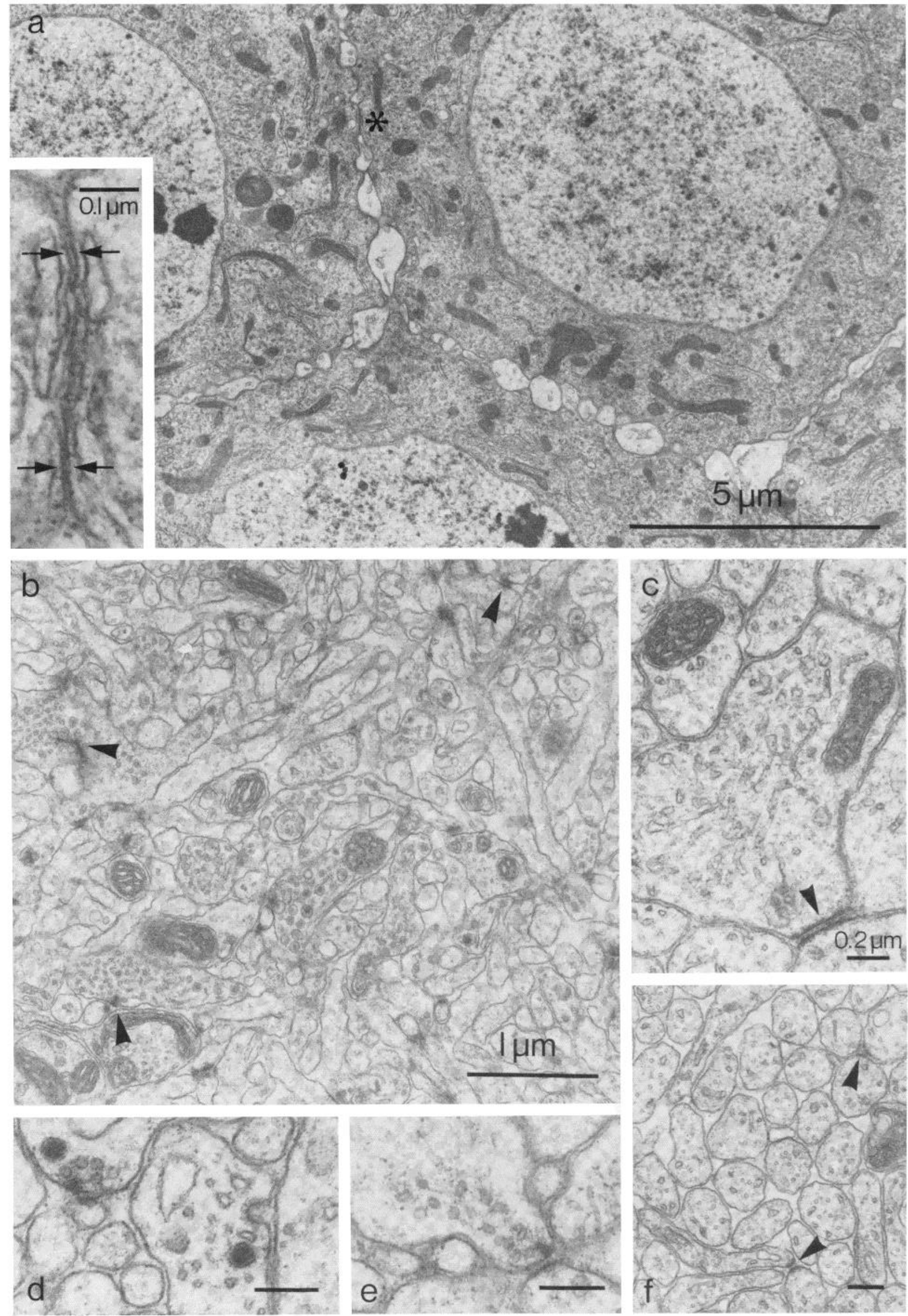

Figure 5. 
not sharply distinguishable from that of the central, nonglomerular neuropil of the antennal lobe. In the glomerular neuropil there are a few thick processes, but most are of very small diameter (approximately 0.1 to 0.3 $\mu \mathrm{m})$ (Fig. $5 b$ ). Most profiles lack organelles other than mitochondria and microtubules. Some of the mediumsized and larger profiles, however, are filled with irregular membranous cisternae or twisted tubules and multivesicular bodies (Fig. $5 c$ ), suggestive of growth cones (Kawana et al., 1971). Coated pits (Fig. 5d) and coated vesicles are seen with much greater frequency than in the mature animal. A few profiles contain synaptic vesicles. At day 5 almost all vesicles have lucent centers, but by day 6 numerous, large, dense-cored vesicles are also present in some of the profiles.

The glomerular neuropil is sprinkled with focal membrane specializations that resemble desmosomes in certain planes of section. The specializations (Fig. $5, b$ and c) consist of membrane-associated densities in two or three adjacent elements, often at the juncture of three elements, and a dense material in the intercellular cleft. The membrane-associated material may be a roughly triangular projection or may be more evenly distributed along the membrane. These two images might represent orthogonal vicws of the same roughly bar-shaped density. At most junctions there are no vesicles in any of the processes contributing specializations; if there are vesicles, they usually are not clustered near the membrane. A few definitive synapses are present, nevertheless, at these early stages. At these synapses (Fig. 5, $d$ and $e$ ), a small number of vesicles clv ter around a presynaptic density that resembles the other membrane-associated densities. The cleft material and postsynaptic densities are indistinguishable from the analogous structures at the nonsynaptic junctions. In a single cross-section there are usually two postsynaptic elements, but whether the total number of postsynaptic elements in a single complex is as high as in the mature animal is not known. The numbers of all membrane specializations and of definite synapses per unit area in thin sections through the glomerular neuropil are plotted in Figure 6 . We did not reconstruct junctions in three dimensions. Thus the sizes of junctions, which would affect the probability of their appearing in a given section, are not known. For simplicity we refer to numbers of junctions, but what may seem to be an increase in number may also reflect a growth of existing junctions-also a significant event-as new cellular elements become joined.

Between profiles in the bundles of neurites coursing through nonglomerular regions of the neuropil, there are occasional symmetrical junctions (Fig. $5 f$ ) like those de-

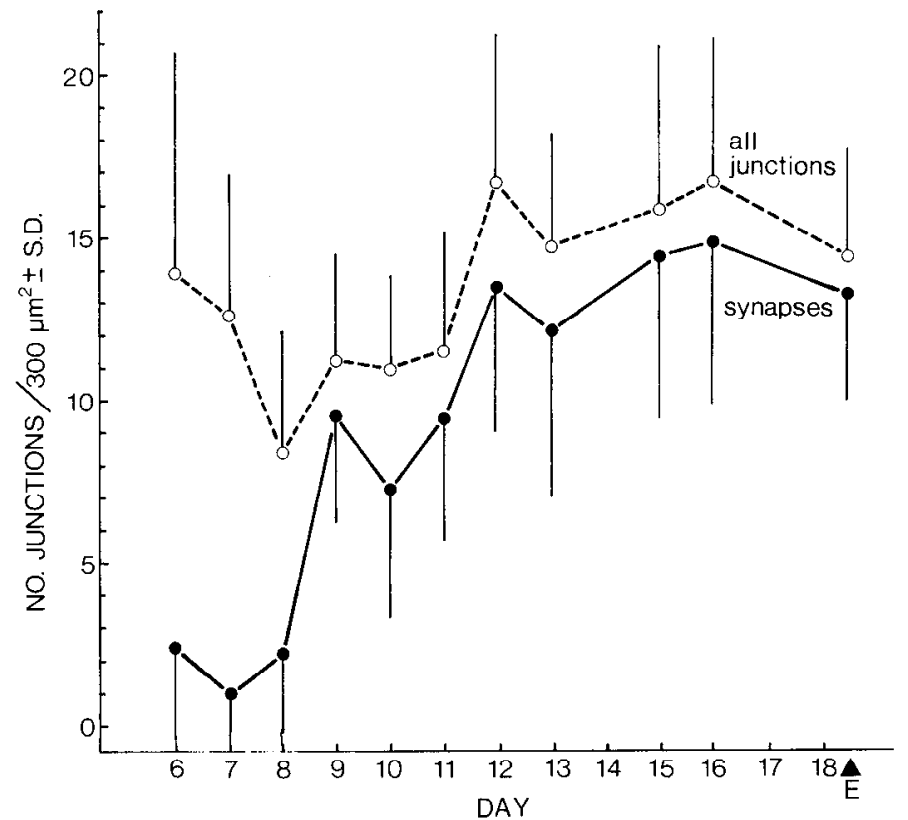

Figure 6 . Number of junctions $/ 300 \mu \mathrm{m}^{2}$ as a function of stage of adult development. $E$ marks the time of eclosion of the adult moth. Junctions were counted in randomly selected electron micrographs each covering $300 \mu \mathrm{m}^{2}$ of glomerular neuropil. At least 20 micrographs were scored for each age except day 6 , for which nine were scored. For days 8 and 10 to 17, micrographs were of material from two to four different brains; for days 6,7 , 9 , and 18 , micrographs were of multiple sections from single brains. Bars indicate the standard deviation for values obtained from all micrographs at a given age. The dotted line plots the packing density of all junctions. The solid line plots that of only synapses; a junction was identified as synaptic if vesicles were clustered near the density of one of the participating membranes.

scribed above. It is not known whether these neurites are axons or whether other parts of the neurons can form bundles at these early stages. In the mature antennal lobe, junctions are never seen between processes that are clearly axons, running in fascicles.

Days 9 through 11. By day 9 the neuronal cell bodies are always separated from each other by several layers of glial processes, as in the adult. In the glomerular neuropil most of the junctions now have vesicles (Fig. 7, $a$ and $c$ ). As shown in Figure 6, although the total number of junctions per unit area has not changed significantly, the percentage of junctions that can clearly be identified as synapses has increased dramatically. Many profiles in the neuropil have begun to expand in diameter and are now filled with either clear, round vesicles or a mixed

Figure 5. Electron micrographs of antennal lobes from animals at days 5 through 8 of adult development. All micrographs were made from material fixed sequentially with aldehydes and then osmium. $a$, Day 8, neuronal cell bodies, whose membranes are apposed at some points but separated by electron-lucent glial processes at others. Inset, Higher magnification of region next to asterisk. Where plasma membranes (arrows) can be resolved from membranes of subsurface cisternae, they appear occasionally to touch. The nature of these possible contacts is not known. $b$, Day 6, glomerular neuropil. Almost all profiles are less than 0.5 $\mu \mathrm{m}$ in diameter. The largest profiles contain small, clear and large, dense-cored vesicles. Junctions (such as those at arrowheads) with associated vesicles or without are scattered throughout. $c$, Day 6 , profile filled with membranous tubules or sacs. This profile, probably representing a growth cone, participates in a symmetrical junction (arrowhead). $S c a l e$ bars for $c$ to $f$ equal $0.2 \mu \mathrm{m}$. $d$, Day 7, synapse. Vesicles cluster around presynaptic density. Postsynaptic densities are not clearly evident in this view. Note coated pit at right. $e$, Day 8, synapse. Presynaptic density is triangular in cross-section. $f$, Day 7 , symmetrical junctions (arrowheads) between neurites traveling in parallel in a bundle. 

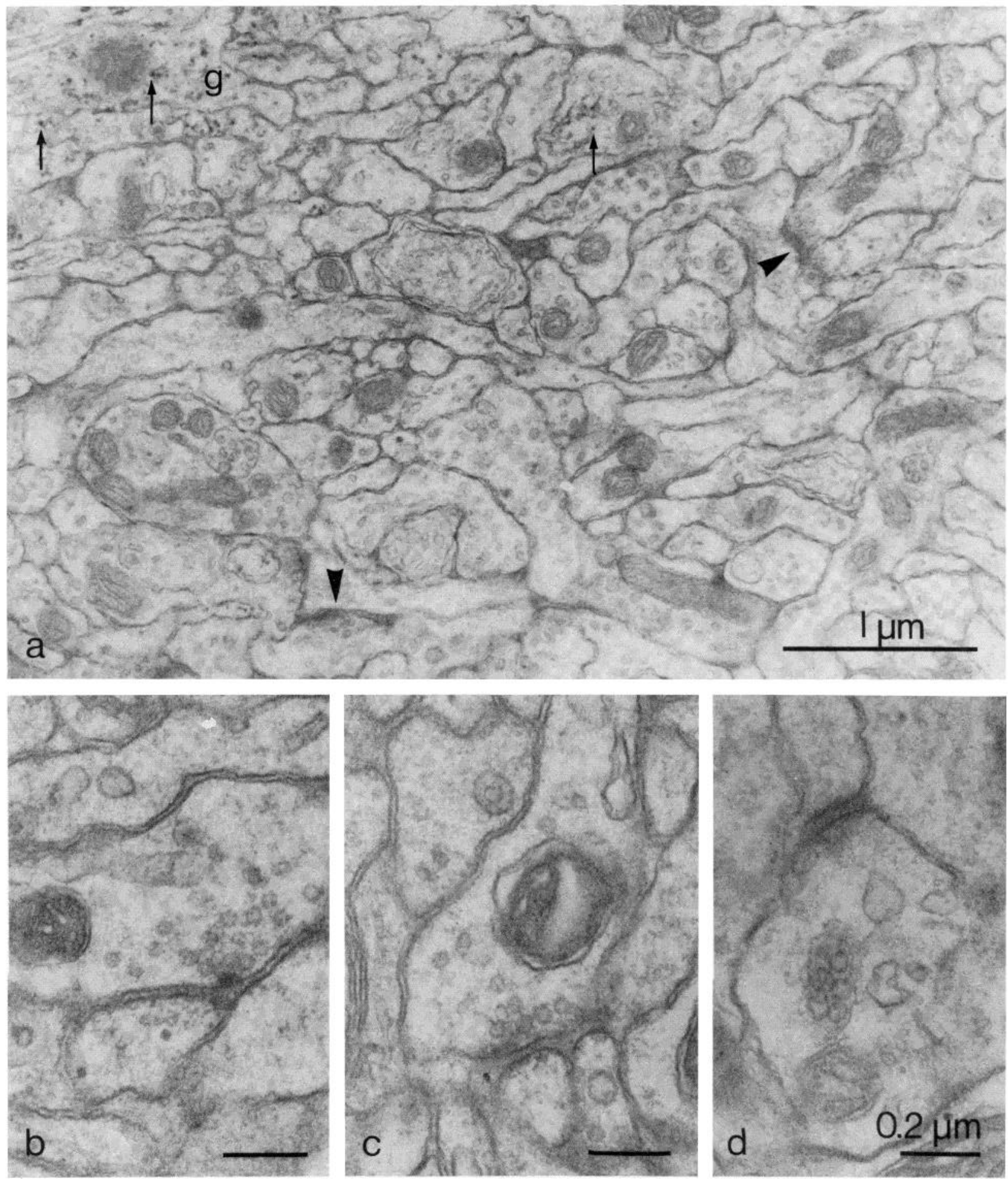

Figure 7. Electron micrographs of glomerular neuropil, day 10. $a$, Edge of a glomerulus, from material simultaneously fixed, osmicated, and stained with lead. Junctions (e.g., at arrowheads) do not show enhanced staining, although glycogen particles (arrows) in glial processes $(g)$ that delimit the glomerulus do, indicating that method has been successful in staining carbohydrate moieties. $b$ and $c$, Synapses from material sequentially fixed and stained. $d$, Symmetrical junction between neurites in same material as that of $b$ and $c$. Scale bars in $b$ to $d$ equal $0.2 \mu \mathrm{m}$.

population of clear, round and large, dense-cored vesicles (not shown). Profiles containing pleiomorphic vesicles have not been identified at early stages; this may indicate that these elements appear late, but more probably it simply reflects their scarcity in adult antennal lobes. In tissue prepared by the method of Simionescu et al. (1972), there is very little membrane-associated material that stains preferentially (Fig. $7 a$ ). In conventionally stained preparations, however, the synapses formed by presynaptic terminals (Fig. 7, $b$ and $c$ ) have most of the characteristics of mature synapses, as seen in single sections: the presynaptic density, triangular or rod-shaped in 
cross-section, has vesicles clustered around it; dense material forms a more or less distinct band in the synaptic cleft; and the postsynaptic elements are lined with densities more extensive than those seen at day 8 . The most obvious difference between these synapses and those in the adult is the variability in the number of vesicles. Although some synapses are identical to those of the adult, many still possess only a few vesicles. A small number of symmetrical membrane-associated densities, like those dominating earlier stages, are still present (Fig. $7 d$ ). Junctions between nonglomerular neuronal processes are no longer seen.

Day 12 through eclosion. By day 12 there are no profiles containing membranous cisternae and tubules that might represent growth cones in the neuropil. Instead, more profiles are filled with synaptic vesicles. Day 12 antennal lobes stained preferentially for membraneassociated densities exhibit numerous, easily identified junctions scattered throughout the glomerular neuropil (Fig. 8a). Most of the junctions are clearly synaptic; the fraction of junctions that are synaptic reaches a plateau at this stage (Fig. 6). Perhaps the few junctions that appear not to include vesicles are actually tangential or peripheral sections through regions of the synapse where there are no accumulations of vesicles. In conventionally stained material, the few symmetrical junctions (e.g., Fig. $8 b$ ) have the same characteristics as at earlier stages, whereas many synapses (Fig. 8, $c$ and $d$ ) now look mature (compare with Fig. 9, $a$ and $b$ ).

The most striking change from day 12 onward is that some profiles continue to grow in diameter until eclosion of the adult moth, when the profiles are up to $2 \mu \mathrm{m}$ across. Tissue from day 16 animals shows that by this stage, synaptic junctions stain very darkly with the leadstaining procedure (Fig. 9c) and are easily recognized even at relatively low magnifications. At higher magnifications (Fig. 9c, inset) comparable to those of Figures $7 a$ and $8 a$, the staining of membrane-associated densities and material in the synaptic cleft almost obscures cellular detail.

Deafferented antennal lobes. Antennal lobes deprived of antennal input throughout adult development are smaller than normal lobes but develop a lobular ring of condensed neuropil that may be thought of as "protoglomerular" (Hildebrand et al., 1979). Electron microscopic examination of chronically deafferented antennal lobes reveals numerous synapses, which are restricted to these protoglomeruli. At day 8 (Fig. 10a), the earliest stage at which we have examined deafferented lobes, the neuropil of the protoglomeruli looks similar to that of the glomeruli of normal antennal lobes of the same age. Most neuritic profiles in the protoglomeruli contain only mitochondria and microtubules, although some contain small round clear vesicles and larger, dense-cored vesicles. Both symmetrical and synaptic junctions are found. As development progresses, more junctions form, and by day 12 (Fig. 10b), almost all of the junctions are clearly synaptic.

\section{Discussion}

During the metamorphosis from larva to moth, antennae arise de novo from imaginal disks. Previous work has shown that all of the olfactory neurons in the antenna of
Manduca sexta are born mitotically on days 1 and 2 of the 18-day adult development and immediately begin to extend axons down the lumen of the antenna (Sanes and Hildebrand, 1976a, b). The axons begin to reach the brain on day 3 , and by day 10 apparently all have arrived (Sanes and Hildebrand, 1976b; Camazine and Hildebrand, 1979). Based on preliminary experiments (K. S. Kent and J. G. Hildebrand, unpublished data) and work on a related species, Danaus plexippus plexippus (Nordlander and Edwards, 1970), it is likely that all neurons in the antennal lobe are born prior to the arrival of the antennal sensory axons in the antennal lobe.

In the present study, we have found that the neuropil of the growing antennal lobe in Manduca appears homogeneous in histological sections until day 5 , when glomeruli develop around the perimeter. By visualization of intracellularly injected cobalt, we have found that neurons of the antennal lobe begin to elaborate neurites during the first half of adult development and continue to sculpture them until about day 12. Chemical synapses can be recognized in thin sections of the antennal lobe as early as day 5, and electrophysiological recordings begin to detect synaptic activity between antennal nerve axons and second-order neurons on day 9. By our criteria, synapses appear to be structurally and physiologically mature by day 13,5 days before the antenna is exposed to the environment and before the electroantennogram indicates that the sensory transduction mechanism begins to function (Schweitzer et al., 1976). Thus all of the development and maturation that we have studied occurs after antennal sensory axons have begun to grow into the antennal lobe but before they can provide input about the environment to the system.

Our findings address several issues in neural development, among which two questions are timely and of general interest. First, what factors could influence the establishment of specialized synaptic regions, the glomeruli, in the neuropil? Second, what is the schedule of events through which interneuronal synapses mature? In particular, how do the structural and functional aspects of synaptogenesis in the antennal lobe correlate with each other?

\section{Formation of glomeruli}

Knowledge of the specific timing of correlated events in a developing system is a first step toward generating testable hypotheses about factors governing the development. In the present study, we have sought to establish the timetable of histological, ultrastructural, and physiological events related to the formation of synapses in the antennal lobes. The separation of the neuropil of the antennal lobes into glomeruli, which may in the adult be specialized to process information about different odors (discussed in Tolbert and Hildebrand, 1981), is a relatively early event.

The glomeruli begin to become distinct from surrounding neuropil on day 5. By this stage in metamorphosis many but not all of the antennal nerve axons have entered the antennal lobe (Sanes and Hildebrand, 1976b; Camazine and Hildebrand, 1979). In Danaus, another lepidopterous insect, Nordlander and Edwards (1970) found that all of the antennal lobe neurons have been born by a comparable stage of adult development. In 

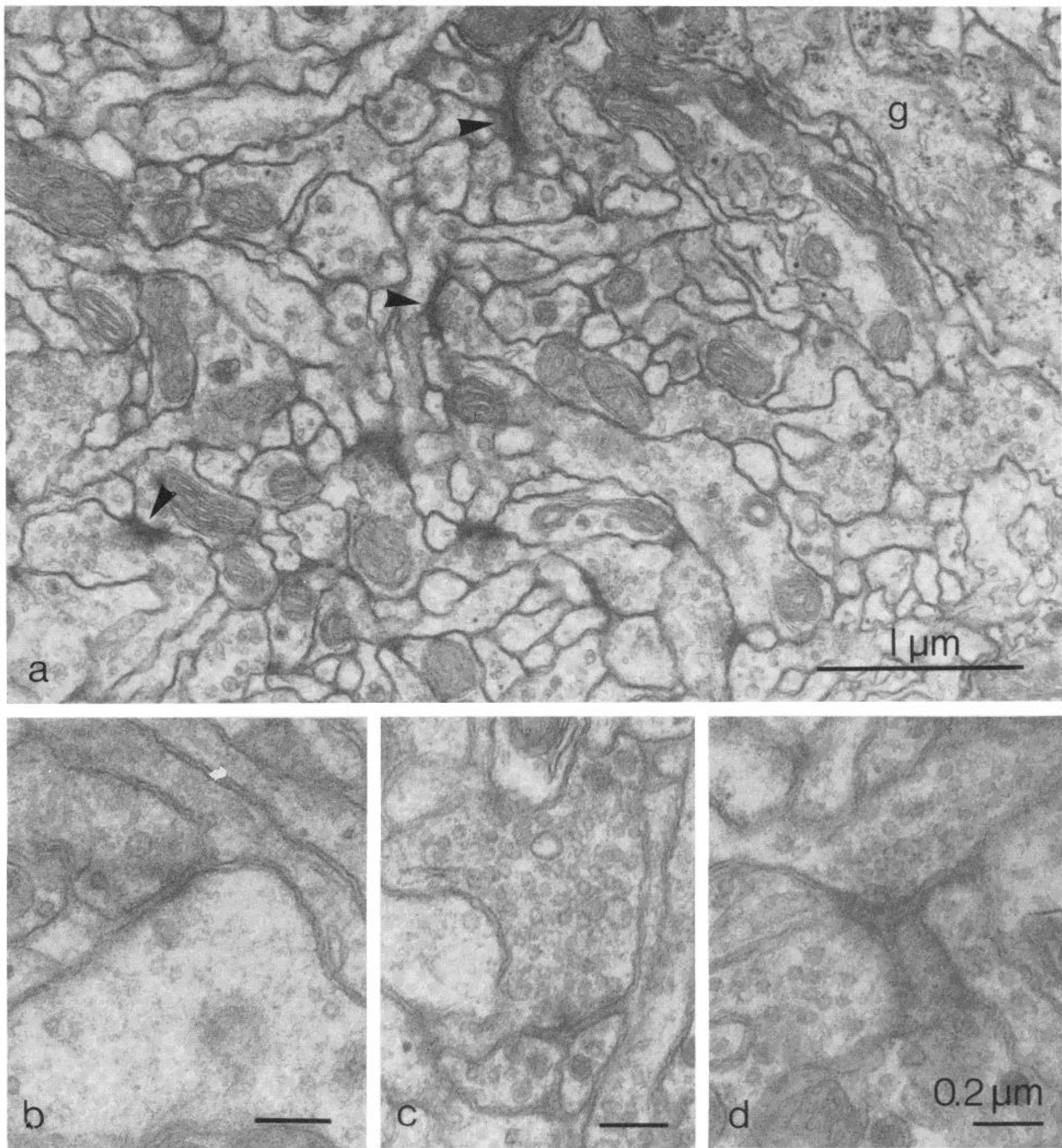

Figure 8. Electron micrographs of glomerular neuropil. $a$, Day 12, edge of a glomerulus, from tissue simultaneously fixed, osmicated, and stained with lead. Junctional specializations (e.g., arrowheads) now stain more densely than any other structures except for glycogen particles, which are concentrated in glial processes $(g)$ bordering the glomerulus. $b$, Day 11 to 12 , symmetrical junction. $c$ and $d$, Day 11 to 12 , synapses in tissue sequentially fixed and stained. Scale bars in $b$ to $d$ equal $0.2 \mu \mathrm{m}$.

Manduca this stage is about 10 days before the olfactory sensilla of the antenna are able to respond to odors even if the antenna is exposed by withdrawal from the surrounding pupal cuticle (Schweitzer et al., 1976). Thus the neuropil becomes glomerular long before afferent information about odors apparently can play a role.

Glomeruli are composed primarily of the terminal branches of the axons of antennal olfactory neurons, the neurites of antennal lobe neurons, and synapses among these elements. The projections of the antennal afferent axons appear in cobalt-filled preparations (S. M. Camazine and J. G. Hildebrand, manuscript in preparation) to be clearly sorted and distinctly glomerular at or soon after the time at which glomeruli begin to be recognizable in histological sections. We have not been able to determine when the neurites of antennal lobe neurons first segregate into glomeruli. By day 8 , the earliest stage at which we have been able to stain the neurons intracellularly, their neurites have already grown into the glomerular neuropil and their branching patterns at least 

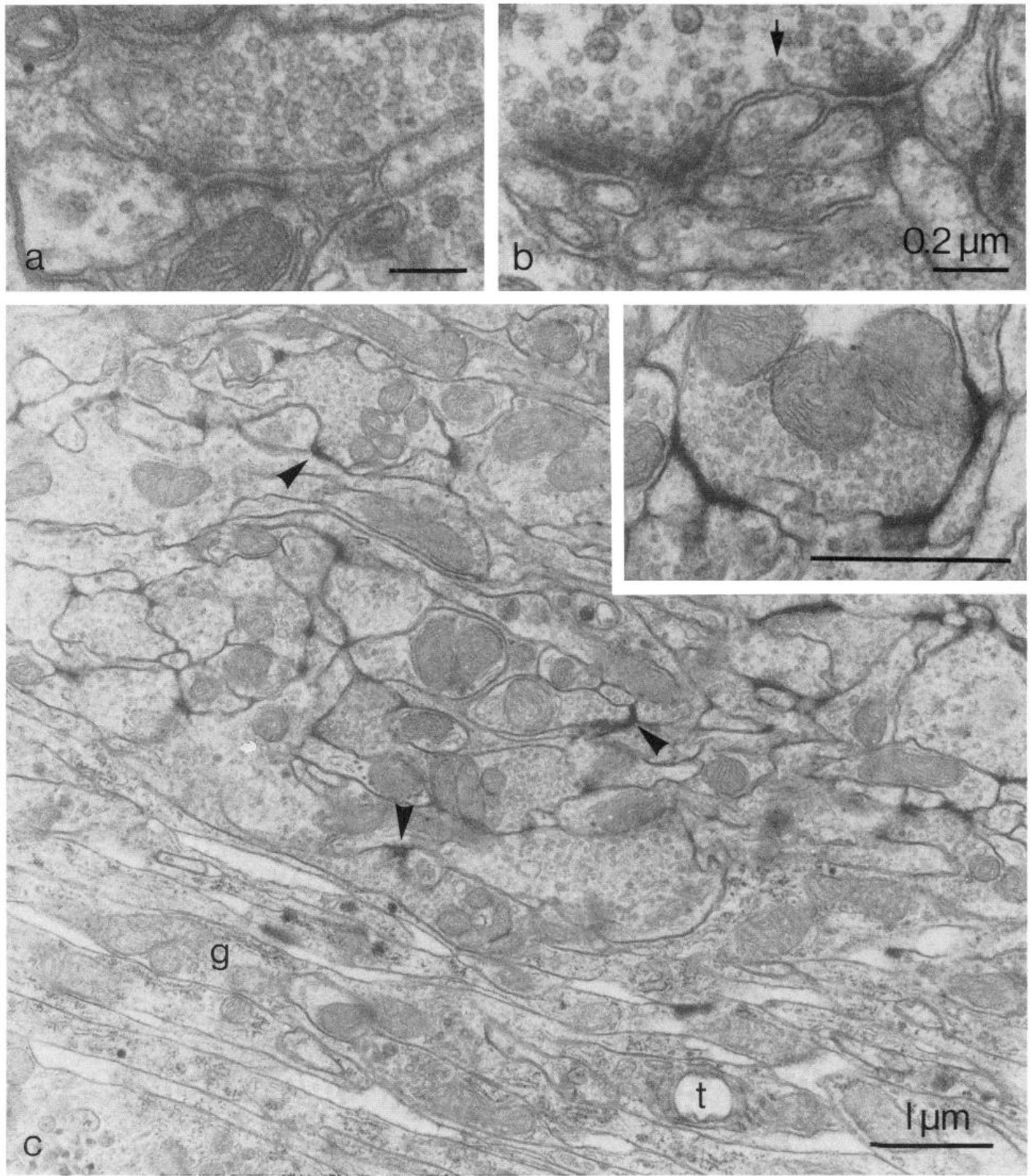

Figure 9. Electron micrographs of glomerular neuropil in mature animals. $a$ and $b$, Adult, synapses from sequentially fixed and stained material. Arrow indicates coated pit between synaptic regions. Scale bars in $a$ and $b$ equal $0.2 \mu \mathrm{m}$. $c$, Day 16, edge of a glomerulus, from material simultaneously fixed, osmicated, and stained with lead. Synapses (e.g., at arrowheads) show enhanced staining as at day 12. Scale bars in large panel and inset equal $1 \mu \mathrm{m}$. Inset is at same magnification as Figures $7 a$ and $8 a$, demonstrating that profiles are continuing to increase in diameter after day $12 . \mathrm{g}$, glial processes; $t$, tracheole.

roughly define glomeruli. The interplay of two mechanisms sculptures the definitive branching patterns of antennal lobe neurons. The type of local interneuron studied in this investigation, characterized in the adult by branches in most or all of the "ordinary" glomeruli, appears to establish its mature branching pattern by continuous embellishment of a primitive scaffold; that is, it lays down a coarse arbor that grows larger and denser through further branching. Some resorption and redirected outgrowth on the finest level, such as that seen by Fröhlich and Meinertzhagen (1980) in the optic lamina of the fly, may occur in the antennal lobe, but these processes have not yet been demonstrated at the light or electron microscopic level. In contrast, the output neu- 

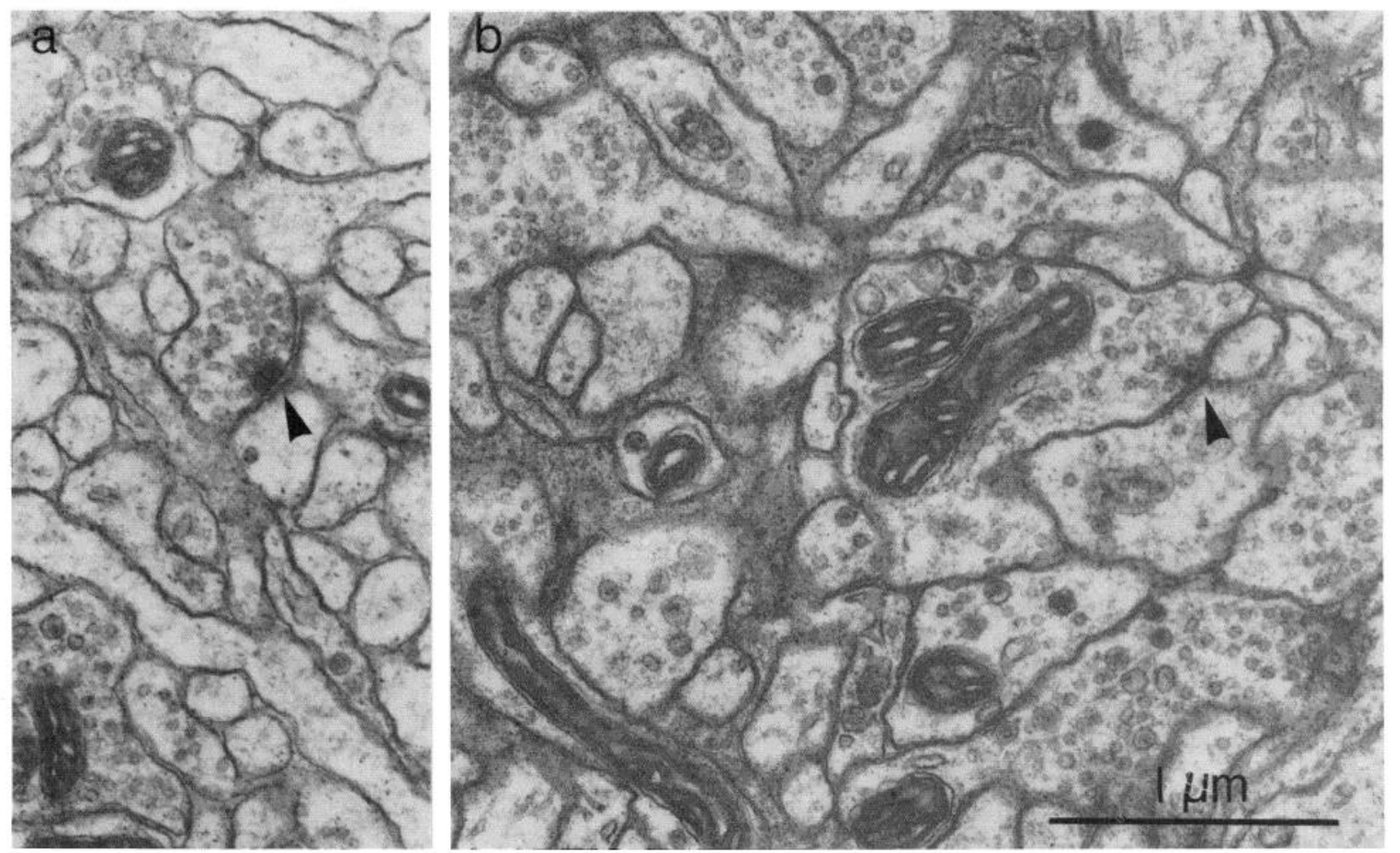

Figure 10. Electron micrographs of glomerular neuropil from deantennated animals, conventionally fixed and stained. Scale bar equals $1 \mu \mathrm{m}$ for $a$ and $b . a$, Day 8, synapse (arrowhead). b, Day 12, synapse (arrowhead).

rons studied here, which are characterized by uniglomerular arbors in the adult, first send out more extensive branches that are subsequently pruned to leave the uniglomerular tuft. Other investigators have shown pruning of central neurons during embryonic development (Wallace, 1980; Goodman et al., 1981) and during metamorphosis (Truman and Reiss, 1976). For technical reasons, we have not been able to observe the morphology of the output neurons before day 9 , when one glomerular branch of the neuritic arbor has already assumed clear dominance. It would be of great interest to know whether the dominant branch is indistinguishable from the rest of the arbor at earlier stages and whether it becomes dominant before histologically conspicuous formation of glomeruli has occurred.

The effects of early removal of the antenna on the histology and $\alpha$-bungarotoxin-binding properties of the antennal lobe have been described (Hildebrand et al., 1979). Deafferented antennal lobes have "protoglomeruli," condensed neuropil structures that roughly resemble glomeruli. Binding of $\alpha$-bungarotoxin, marking putative postsynaptic sites, is restricted to the protoglomeruli, just as it is restricted to the glomeruli in the normal animal. In the present study, we confirm by electron microscopy that synapses in the deafferented antennal lobe are restricted to the protoglomeruli. The possible identity of these synapses is discussed in the next section. It appears that antennal nerve input is not necessary for the segregation of synaptic and nonsynaptic regions of the antennal lobe. Moreover, we have not yet found other inputs to the antennal lobe that could provide an organizing influence for development of protoglomeruli. On the other hand, antennal nerve input is necessary for the establishment of distinct glomeruli, surrounded by glial cells.

\section{Synaptic development}

Correlations between physiology and anatomy. The temporal relationship between functional maturation and morphogenesis of synapses in the antennal lobe is emerging but is still difficult to interpret. In the present study, we have found that synapses become recognizable in the electron microscope at least 3 days before synaptic potentials can be monitored electrophysiologically in response to stimulation of the antennal nerve. Previous studies in our laboratory have shown that acetylcholine, which is apparently the neurotransmitter of antennal sensory neurons (Sanes and Hildebrand, 1976c), and related enzymes reach detectable levels in the antenna on day 4, 1 day after the sensory neurons are born. These levels reach adult values in the antenna between days 13 and 16 and rise in an intracranial segment of the antennal nerve at about the same time, indicating that the sensory axons can transport neurotransmitter machinery toward their terminals at an early stage in their development (Sanes and Hildebrand, 1976c). Thus, we thought that antennal nerve axons might have the ability to release transmitter at early stages. Spontaneous release of neurotransmitter may begin to occur at an early stage of synapse formation, although our recording techniques did not routinely allow us to investigate that important question. In addition, numbers of $\alpha$-bungarotoxin-binding sites in the glomeruli rise gradually throughout metamorphosis (Hildebrand et al., 1979), suggesting that at least some postsynaptic sites might mature early. The 
results of the present study, however, suggest that the antennal sensory axons may release little or no neurotransmitter, or that the postsynaptic neurons do not respond appreciably to released transmitter, until about halfway through adult development.

Correlated anatomical and physiological studies of the developing neuromuscular junction in Xenopus (Kullberg et al., 1977), synapses in spinal cord cultures (Crain and Peterson, 1967), and synapses in the ciliary ganglion of the chick (Landmesser and Pilar, 1972) have suggested that the onset of synaptic transmission precedes the appearance of anatomically recognizable synaptic junctions. This has led one group of authors (Kullberg et al., 1977) to conclude that the earliest contacts made by neuronal processes capable of releasing transmitter are morphologically undifferentiated. In the present case, anatomical evidence of synapses in the antennal lobe precedes physiological evidence of transmission from the antennal nerve. This would easily be explained if the earliest synaptic contacts in the antennal lobe are lateral interactions between antennal lobe neurons or contacts by descending elements (as yet unidentified) from higher centers in the brain, a suggestion consistent with the finding that deafferented antennal lobes begin to display synapses at about the same time as normal lobes. On the other hand, it could be that the early synapses in normal antennal lobes are made by antennal nerve axons and that a minimum number of these synapses must simultaneously affect the postsynaptic neuron before one can record postsynaptic activity in the main neurite or cell body. Sparse distribution of presynaptic terminals or altered electrotonic properties of the postsynaptic cell would clearly affect this threshold. Despite repeated attempts to fill sensory axons and second-order neurons in immature animals with horseradish peroxidase, we have not been able to distinguish in a direct way which cellular elements in the antennal lobe are forming the earliest synapses.

Other than our own, the only information of which we are aware that correlates the light and electron microscopic anatomy and electrophysiology of synapses maturing during metamorphosis comes from the work of Stocker and Nüesch (1975; Nüesch, 1965), Heinertz (1976), and Kaufmann (1971) on the innervation of flight muscle in the moth Antheraea polyphemus. Soon after pupal ecdysis in Antheraea, the distal portions of the motor nerves in certain pupal muscles degenerate and the nerves sprout to form new connections with flight muscle myotubes. At first nerve and muscle membranes lie next to each other, forming no specializations, but even before that the axons have finished growing, many presynaptic and a few postsynaptic specializations appear. These early junctions are believed to disappear, and new synapses start to form on day 10 of the 21-day adult development. Nüesch (1965) reported that strong ganglionic stimulation can produce contractions in the muscle starting on day 10, whereas Kaufmann (1971) was unable to detect endplate potentials until day 13. Synaptic contacts look almost mature by day 14, but even at day 16 Nüesch found evidence that the physiological properties of the system might not yet be mature. The discrepancy between the physiological results at a critical stage must be resolved before one can identify what structural features underlie the earliest functional contacts in that system.

Ultrastructural development of synapses. In the present study we have counted the symmetrical and synaptic contacts in the glomerular neuropil of the antennal lobe at various stages of metamorphic adult development. We have found that the packing density of junctions changes little at least from day 6 onward. Because the volume of neuropil increases with age until about day 11, it seems that the absolute number of junctions increases. At early times, symmetrical contacts that resemble desmosomes predominate, but by day 15 almost all junctions are unequivocally synapses. The remaining junctions, identified as symmetrical membrane-associated densities in two or three abutting processes, may be nonsynaptic or may be tangential or peripheral sections through synapses.

Our findings contribute to the large fund of data from vertebrate material (e.g., Bunge et al., 1967; Masurovsky et al., 1971; Hinds and Hinds, 1976) suggesting that desmosome-like structures play a role in synaptogenesis, but they do not help to close the debate on whether these junctions perform a temporary adhesive role or represent the earliest structural parts of synapses. We use the term "desmosome-like structures" because, until the molecular structures of these symmetrical junctions in vertebrates and invertebrates are compared with the molecular structure of the desmosome, one cannot conclude that the junctions perform the same (adhesive) function simply on anatomical grounds. In other invertebrates as well, symmetrical specializations have been observed in developing neuropil. As in Manduca, the specializations occur both at places where synapses will not form (TrujilloCenóz and Melamed, 1973; LoPresti et al., 1974; Lane and Swales, 1979) and in regions where synapses are destined to form (Trujillo-Cenóz and Melamed, 1973; Lane and Swales, 1979).

Synapses in the antennal lobe mature by a gradual accretion of vesicles and a gradual thickening and increase in density of membrane-associated material, as has also been noted in many vertebrate systems (Aghajanian and Bloom, 1967; Masurovsky et al., 1971; Hinds and Hinds, 1976; Rebière and Dainat, 1981). In the optic lamina of the fly (Fröhlich and Meinertzhagen, 1982) during the last $20 \%$ of pupal development, the number of presynaptic vesicles increases, and the shape and staining characteristics of the presynaptic bar change. In the fly the number of postsynaptic elements also increases. It is not known whether such an enlargement of synapses occurs in the antennal lobe of Manduca.

Larramendi (1969), in the cerebellum of the mouse, and Fröhlich and Meinertzhagen (personal communication), in the optic lamina of the fly, have found evidence for an overproduction of synapses followed by a subsequent decrease in their number during development. Such a process might provide a mechanism for fine tuning of synaptic connections. The data in Figure 6 show no evidence for such a process in developing antennal lobes, but counts of total synapses would not reveal it if new synapses were laid down at least as fast as other synapses were culled. The simplest interpretation of the present observations is that there is a progressive establishment of new synapses, with no destruction of syn- 
apses once they have formed, but this interpretation requires further evidence.

The present study has revealed the temporal relationships between some of the processes important in the development of appropriate synaptic contacts in a complex network, the antennal lobe of Manduca sexta. These temporal relationships provide insights into the influence of sensory axons on postsynaptic neurons and provide important guidelines for future experimentation.

\section{References}

Aghajanian, G. K., and F. E. Bloom (1967) The formation of synaptic junctions in developing rat brain: $A$ quantitative electron microscopic study. Brain Res. 6: 716-727.

Anderson, H. (1978) Postembryonic development of the visual system of the locust, Schistocerca gregaria, II. An experimental investigation of the formation of the retina-lamina projection. J. Embryol. Exp. Morphol. 46: 147-170.

Bacon, J. P., and J. S. Altman (1977) A silver intensification method for cobalt-filled neurones in wholemount preparations. Brain Res. 138: 359-363.

Black, I. B., I. A. Hendry, and L. L. Iversen (1971) Transsynaptic regulation of growth and development of adrenergic neurons in the mouse sympathetic ganglion. Brain Res. 34: 229240.

Black, I. B., I. A. Hendry, and L. L. Iversen (1972) Role of postsynaptic neurons in the biochemical maturation of presymaptic cholinergic nerve terminals in a mouse sympathetic ganglion. J. Physiol. (Lond.) 221: 149-159.

Bloom, F. E., and Aghajanian, G. K. (1968) Fine structural and cytochemical analysis of the staining of synaptic junctions with phosphotungstic acid. I. Ultrastruct. Res. 22: 361-375.

Bunge, M. B., R. R. Bunge, and E. R. Peterson (1967) Onset of synapse formation in spinal cord cultures as studied by electron microscopy. Brain Res. 6: 728-749.

Camazine, S. M., and J. G. Hildebrand (1979) Central projections of antennal sensory neurons in mature and developing Manduca sexta. Soc. Neurosci. Abstr. 5: 155.

Cowan, W. M., and E. Wenger (1967) Cell loss in trochlear nucleus of chick during normal development and after radical extirpation of the optic vesicle. J. Exp. Zool. 164: 267-280.

Crain, S. M., and E. R. Peterson (1967) Onset and development of functional interneuronal connections in explants of rat spinal cord-ganglia during maturation in culture. Brain Res. 6: 750-762.

Fröhlich, A., and I. A. Meinertzhagen (1980) Neurite growth and its relationship to synaptogenesis in the fly's visual system. Soc. Neurosci. Abstr. 6: 373.

Fröhlich, A., and I. A. Meinertzhagen (1982) Synaptogenesis in the first optic neuropile of the fly's visual system. J. Neurocytol. 11: 159-180.

Goodman, C. S., M. Bate, and N. C. Spitzer (1981) Embryonic development of identified neurons: Origin and transformation of the H cell. J. Neurosci. 1: 94-102.

Guth, L. (1957) Effects of glossopharyngeal nerve transsection on the circumvallate papilla of the rat. Anat. Rec. 28: 715731.

Heinertz, R. (1976) Untersuchungen am thorakalen nervensystem von Antheraea polyphemus Cr. (Lepidoptera) unter besonderer Berücksichtigung der Metamorphose. Rev. Suisse Zool. 83: 215-242.

Hildebrand, J. G., L. M. Hall, and B. C. Osmond (1979) Distribution of binding sites for ${ }^{125}$ I-labeled $\alpha$-bungarotoxin in normal and deafferented antennal lobes of Manduca sexta. Proc. Natl. Acad. Sci. U. S. A. 76: 499-503.

Hinds, J. W., and P. L. Hinds (1976) Synapse formation in the mouse olfactory bulb. II. Morphogenesis. J. Comp. Neurol. 169: 41-62.

Karnovsky, M. J. (1965) A formaldehyde-glutaraldehyde fixative of high osmolality for use in electron-microscopy. J. Cell Biol. 27: 137A-138A.

Kaufmann, L. (1971) Untersuchungen zur Funktion der Flugmuskulatur von Antherea polyphemus (Lep.) während der Imaginalentwicklung. Rev. Suisse Zool. 78: 1007-1036.

Kawana, E., C. Sandri, and K. Akert (1971) Ultrastructure of growth cones in the cerebellar cortex of the neonatal rat and cat. Z. Zellforsch. 115: 284-298.

Kopeć, S. (1922) Mutual relationship in the development of the brain and eyes of Lepidoptera. J. Exp. Zool. 36: 459-468.

Kullberg, R. W., T. L. Lentz, and M. W. Cohen (1977) Development of the myotomal neuromuscular junction in Xenopus laevis: An electrophysiological and fine-structural study. Dev. Biol. 60: 101-129.

Landmesser, L., and G. Pilar (1972) The onset and development of transmission in the chick ciliary ganglion. J. Physiol. (Lond.) 222: 691-713.

Lane, N. J., and L. S. Swales (1979) Intercellular junctions and the development of the blood-brain barrier in Manduca sexta. Brain Res. 168: 227-245.

Larramendi, L. M. H. (1969) Analysis of synaptogenesis in the cerebellum of the mouse. In Nearobiology of Cerebellar Evolution and Development, R. Llinas, ed., pp. 803-843, American Medical Association, Chicago.

LoPresti, V., E. R. Macagno, and C. Levinthal (1974) Structure and development of neuronal connections in isogenic organisms: Transient gap junctions between growing optic axons and lamina neuroblasts. Proc. Natl. Acad. Sci. U. S. A. 71: 1098-1102.

Luna, L. G. (1968) Manual of Histologic Staining Methods of the Armed Forces Institute of Pathology, McGraw-Hill, New York.

Macagno, E. R. (1977) Abnormal synaptic connectivity following UV-induced cell death during Daphnia development. In Cell and Tissue Interactions, J. W. Lash and M. M. Burger, eds., pp. 293-309, Raven Press, New York.

Masurovsky, E. B., H. H. Benitez, and M. R. Murray (1971) Synaptic development in long-term organized cultures of murine hypothalamus. J. Comp. Neurol. 143: 263-278.

Matsumoto, S. G., and J. G. Hildebrand (1981) Olfactory mechanisms in the moth Manduca sexta: Response characteristics and morphology of central neurons in the antennal lobes. Proc. R. Soc. Lond. (Biol.) 213: 249-277.

Maxwell, G. D., and J. G. Hildebrand (1981) Anatomical and neurochemical consequences of deafferentation in the development of the visual system of the moth Manduca sexta. J. Comp. Neurol. 195: 667-680.

Nair, K. M. (1963) Occurrence of anucleolate oocytes in Chrysocaris purpureus (Westw.). Exp. Cell Res. 30: 431-432.

Nordlander, R. H., and J. S. Edwards (1970) Postembryonic brain development in the Monarch butterfly, Danaus plexippus plexippus L. III. Morphogenesis of centers other than the optic lobes. Wilhelm Roux' Arch. 164: 247-260.

Nüesch, H. (1965) Über die strukturelle und funktionelle Entwicklung der Muskeln bei Antherea (Lepidoptera). Z. Naturforsch. 206: 343-347.

Pfenninger, K. H. (1971) The cytochemistry of synaptic densities. (1) An analysis of the bismuth iodide impregnation method. J. Ultrastruct. Res. 34: 103-122.

Rebière, A., and J. Dainat (1981) Quantitative study of synapse formation in the duck olfactory bulb. J. Comp. Neurol. 203: 103-120.

Sanes, J. R., and J. G. Hildebrand (1976a) Structure and development of antennae in a moth, Manduca sexta. Dev. Biol. 51: 282-299. 
Sanes, J. R., and J. G. Hildebrand (1976b) Origin and morphogenesis of sensory neurons in an insect antennae. Dev. Biol. 51: $300-319$.

Sanes, J. R., and J. G. Hildebrand (1976c) Acetylcholine and its metabolic enzymes in developing antennae of the moth, $\mathrm{Man}$. duca sexta. Dev. Biol. 52: 105-120.

Sanes, J. R., J. G. Hildebrand, and D. J. Prescott (1976) Differentiation of insect sensory neurons in the absence of their normal synaptic targets. Dev. Biol. 52: 121-127.

Sanes, J. R., D. J. Prescott, and J. G. Hildebrand (1977) Cholinergic neurochemical development of normal and deafferented antennal lobes during metamorphosis of the moth, Manduca sexta. Brain Res. 119: 389-402.

Schneiderman, A. M., S. G. Matsumoto, and J. G. Hildebrand (1982) Trans-sexually grafted antennae influence development of sexually dimorphic neurones in moth brain. Nature 298: 844-846.

Schweitzer, E. S., J. R. Sanes, and J. G. Hildebrand (1976) Ontogeny of electroantennogram responses in the moth, Manduca sexta. J. Insect. Physiol. 22: 955-960.

Simionescu, N., M. Simionescu, and G. E. Palade (1972) Perme- ability of intestinal capillaries. Pathways followed by dextrans and glycogens. J. Cell Biol. 53: 365-392.

Stocker, R. F., and H. Nüesch (1975) Ultrastructural studies on neuromuscular contacts and the formation of junctions in the flight muscle of Antherea polyphemus (Lep.) I. Normal adult development. Cell Tissue Res. 159: 245-266.

Tolbert, L. P., and J. G. Hildebrand (1981) Organization and synaptic ultrastructure of glomeruli in the antennal lobes of the moth Manduca sexta: A study using thin sections and freeze-fracture. Proc. R. Soc. Lond. (Biol.) 213: 279-301.

Trujillo-Cenóz, O., and J. Melamed (1973) The development of the retina-lamina complex in muscoid flies. J. Ultrastruct. Res. 42: 554-581.

Truman, J. W., and S. E. Reiss (1976) Dendritic reorganization of an identified motorneuron during metamorphosis of the tobacco hornworm moth. Science 192: 477-479.

Venable, J., and R. Coggeshall (1965) A simplified lead citrate stain for use in electron microscopy. J. Cell Biol. 25: 407-408.

Wallace, B. G. (1980) Selective neurite atrophy during development of cells in the leech CNS. Soc. Neurosci. Abstr. 6: 679. 